Bull. Korean Math. Soc. 51 (2014), No. 3, pp. 621-640

http://dx.doi.org/10.4134/BKMS.2014.51.3.621

\title{
SOLUTIONS OF SYSTEMS OF VARIATIONAL INEQUALITIES ON FIXED POINTS OF NONEXPANSIVE MAPPINGS
}

\author{
HOSSEIN PIRI
}

\begin{abstract}
In this paper, we introduce a new approximating method for finding the common element of the set of fixed points of nonexpansive mappings and the set of solution of system variational inequalities for finite family of inverse strongly monotone mappings and strictly pseudocontractive of Browder-Petryshyn type mappings. We show that the sequence converges strongly to a common element the above two sets under some parameter controling conditions. Our results improve and extend the results announced by many others.
\end{abstract}

\section{Introduction}

Let $C$ be a nonempty closed convex subset of a real Hilbert space $H$ and let $A: C \rightarrow H$ be a nonlinear mapping. The classical variational inequality problem is to fined $x \in C$ such that

$$
\langle A x, y-x\rangle \geq 0, \quad \forall y \in C .
$$

The set of solution of (1) is denoted by $\operatorname{VI}(C, A)$, that is,

$$
V I(C, A)=\{x \in C:\langle A x, y-x\rangle \geq 0, \quad \forall y \in C\} .
$$

The variational inequality has been extensively studied in the literature. See e.g. $[5,6,7,9]$ and the references therein. A mapping $A: C \rightarrow H$ is called $\delta$-inverse strongly monotone if there exists a positive real number $\delta>0$ such that

$$
\langle A x-A y, x-y\rangle \geq \delta\|A x-A y\|^{2}, \quad \forall x, y \in C .
$$

It is obvious that any $\alpha$-inverse strongly monotone mapping is monotone and Lipschitzian. A mapping $T: C \rightarrow H$ is called nonexpansive

$$
\|T x-T y\| \leq\|x-y\|, \quad \forall x, y \in C .
$$

Received May 9, 2012; Revised July 3, 2012.

2010 Mathematics Subject Classification. 20D15, 20 D45.

Key words and phrases. fixed point, $\delta$-strongly monotone, $\lambda$-strictly pseudo-contractive, strong convergence, nonexpansive mapping. 
We denote by $\operatorname{Fix}(T)$ the set of fixed point of $T$. Recall that a self-mapping $f: C \rightarrow C$ is a contraction on $C$ if there exists a constant $\alpha \in(0,1)$ such that

$$
\|f(x)-f(y)\| \leq \alpha\|x-y\|, \quad \forall x, y \in C .
$$

For each $x \in H$, there exists a unique nearest point in $C$, denoted by $P_{C} x$ such that

$$
\left\|x-P_{C} x\right\| \leq\|x-y\|, \quad \forall y \in C .
$$

$P_{C}$ is called the projection of $H$ onto $C$.

For finding an element of $F i x(T) \cap V I(C, A)$, Chen et al. [4] introduced the following iterative scheme:

$$
x_{n+1}=\alpha_{n} f\left(x_{n}\right)+\left(1-\alpha_{n}\right) T P_{C}\left(I-\lambda_{n} A\right) x_{n},
$$

where $\left\{\alpha_{n}\right\}$ and $\left\{\lambda_{n}\right\}$ are chosen so that $\lambda_{n} \in[a, b]$ for some $a, b$ with $0<a<$ $b<2 \delta$ and

$\lim _{n \rightarrow \infty} \alpha_{n}=0, \quad \sum_{n=1}^{\infty} \alpha_{n}=\infty, \quad \sum_{n=1}^{\infty}\left|\alpha_{n+1}-\alpha_{n}\right|<\infty, \quad \sum_{n=1}^{\infty}\left|\lambda_{n+1}-\lambda_{n}\right|<\infty$.

They prove that the sequence $\left\{x_{n}\right\}_{n=1}^{\infty}$ converges strongly to $x^{*} \in F i x(T) \cap$ $V I(C, A)$, which is the unique solution of the variational inequality:

$$
\left\langle(I-f) x^{*}, x-x^{*}\right\rangle \geq 0, \quad \forall x \in F i x(T) \cap V I(C, A) .
$$

On the other hand, Yao and Yao [21] introduced the following iterative process: $x_{1}=u \in C$

$$
\left\{\begin{array}{l}
y_{n}=P_{C}\left(I-\lambda_{n} A\right) x_{n} \\
x_{n+1}=\alpha_{n} u+\beta_{n} x_{n}+\gamma_{n} T P_{C}\left(I-\lambda_{n} A\right) y_{n}
\end{array}\right.
$$

where $\left\{\alpha_{n}\right\},\left\{\beta_{n}\right\},\left\{\gamma_{n}\right\}$ and $\left\{\lambda_{n}\right\}$ are chosen so that $\lambda_{n} \in[a, b]$ for some $a, b$ with $0<a<b<2 \delta$ and

$$
\begin{aligned}
& \left(B_{1}\right) \alpha_{n}+\beta_{n}+\gamma_{n}=1, \\
& \left(B_{2}\right) \lim _{n \rightarrow \infty} \alpha_{n}=0, \sum_{n=1}^{\infty} \alpha_{n}=\infty, \\
& \left(B_{3}\right) 0<\liminf _{n \rightarrow \infty} \lambda_{n} \leq \limsup _{n \rightarrow \infty} \lambda_{n}<1, \\
& \left(B_{4}\right) \lim _{n \rightarrow \infty}\left(\lambda_{n+1}-\lambda_{n}\right)=0 .
\end{aligned}
$$

Then $\left\{x_{n}\right\}_{n=1}^{\infty}$ converges strongly to $P_{F i x(T) \cap V I(C, A)} u$.

Recall that a self-mapping $T: C \rightarrow C$ is called $\lambda$-strictly pseudo-contractive of Browder and Petryshyn type [2] if there exists a constant $\lambda \in(0,1)$ such that

(2) $\quad\|T x-T y\|^{2} \leq\|x-y\|^{2}+\lambda\|(I-T) x-(I-T) y\|^{2}, \quad \forall x, y \in C$.

It is well-known that the last inequality is equivalent to

$$
\langle T x-T y, x-y\rangle \leq\|x-y\|^{2}-\frac{1-\lambda}{2}\|(I-T) x-(I-T) y\|^{2}, \quad \forall x, y \in C .
$$


In this paper, motivated and inspired by the iterative schemes considered in $[3,4,12,14,15,16,17,19,21]$, we introduce the iterative below, with the initial guess $x_{0} \in C$ chosen arbitrarily,

$$
\begin{aligned}
& x_{n+1}= \alpha_{n} \gamma f\left(T P_{C}\left(I-\delta_{1, n} A_{1}\right) y_{1, n}\right)+\beta_{n} P_{C}\left(I-\delta_{2, n} A_{2}\right) x_{n} \\
&+\left(\left(1-\beta_{n}\right) I-\alpha_{n} F\right) T P_{C}\left(I-\delta_{3, n} A_{3}\right) y_{1, n}, \\
& y_{i, n}=\gamma_{i, n} P_{C}\left(I-\delta_{i, n} A_{i}\right) y_{i+1, n}+\gamma_{i+1, n} P_{C}\left(I-\delta_{i+1, n} A_{i+1}\right) y_{i+1, n} \\
& \quad+\gamma_{i+2, n} P_{C}\left(I-\delta_{i+2, n} A_{i+2}\right) y_{i+1, n}, \quad i=1,2, \ldots, m, \\
& y_{m+1, n}=x_{n},
\end{aligned}
$$

where $P_{C}$ is a metric projection of $H$ onto $C$ for $i=1,2 \ldots, m, A_{i}: C \rightarrow H$ is a $\delta_{i}$-inverse strongly monotone mapping, $F: C \rightarrow C$ is a mapping which is both $\delta$-strongly monotone and $\lambda$-strictly pseudo-contractive of Browder-Petryshyn type such that $\delta>\frac{1+\lambda}{2}, f$ is a contraction on $H$ with coefficient $0<\alpha<1$ and $\gamma$ is a positive real number such that $\gamma<\left(1-\sqrt{\frac{2-2 \delta}{1-\lambda}}\right) / \alpha$. Our purpose in this paper is to introduce this general iterative algorithm for approximating a fixed point of nonexpansive mappings and the solution set of the system of variational inequalities for finite family of $\delta$-invers strongly monotone mappings and $\lambda$-strictly pseudo-contractive mappings of Browder-Petryshyn. We remark that our results improve and extend the results of Marino and $\mathrm{Xu}$ [10], Yao and Yao [21], Chen et al. [4] and many others.

\section{Preliminaries}

This section collects some lemmas which will be used in the proofs of the main results in the next section.

Lemma 2.1 ([5]). For a given $x \in H, y \in C$,

$$
y=P_{C} x \Leftrightarrow\langle y-x, z-y\rangle \geq 0, \quad \forall z \in C .
$$

It is well known that $P_{C}$ is a firmly nonexpansive mapping of $H$ onto $C$ and satisfies

$$
\left\|P_{C} x-P_{C} y\right\|^{2} \leq\left\langle P_{C} x-P_{C} y, x-y\right\rangle, \quad \forall x, y \in H .
$$

Moreover, $P_{C}$ is characterized by the following properties: $P_{C} x \in C$ and for all $x \in H, y \in C$,

$$
\left\langle x-P_{C} x, y-P_{C} x\right\rangle \leq 0 .
$$

It is easy to see that (4) is equivalent to the following inequality

$$
\|x-y\|^{2} \geq\left\|x-P_{C} x\right\|^{2}+\left\|y-P_{C} x\right\|^{2} .
$$

Using Lemma 2.1, one can see that the variational inequality (1) is equivalent to a fixed point problem.

It is easy to see that the following is true:

$$
u \in V I(C, A) \Leftrightarrow u=P_{C}(u-\lambda A u), \quad \lambda>0 .
$$


A set-valued mapping $U: H \rightarrow 2^{H}$ is called monotone if for all $x, y \in H, f \in$ $U x$ and $g \in U y$ imply $\langle x-y, f-g\rangle \geq 0$. A monotone mapping $U: H \rightarrow 2^{H}$ is maximal if the graph of $G(U)$ of $U$ is not properly contained in the graph of any other monotone mapping. It is known that a monotone mapping $U$ is maximal if and only if for $(x, f) \in H \times H,\langle x-y, f-g\rangle \geq 0$ for every $(y, g) \in G(U)$ implies that $f \in U x$. Let $A$ be a monotone mapping of $C$ into $H$ and let $N_{C} x$ be the normal cone to $C$ at $x \in C$, that is, $N_{C} x=\{y \in H:\langle z-x, y\rangle \leq 0, \forall z \in C\}$ and define

$$
U x= \begin{cases}A x+N_{C} x, & x \in C, \\ \emptyset & x \notin C .\end{cases}
$$

Then $U$ is the maximal monotone and $0 \in U x$ if and only if $x \in V I(C, A)$; see [18].

Let $C$ be a nonempty subset of a Hilbert space $H$ and $T: C \rightarrow H$ a mapping. Then $T$ is said to be demiclosed at $v \in H$ if, for any sequence $\left\{x_{n}\right\}$ in $C$, the following implication holds:

$$
x_{n} \rightarrow u \in C \quad \text { and } \quad T x_{n} \rightarrow v \quad \text { imply } \quad T u=v,
$$

where $\rightarrow$ (resp. $\rightarrow$ ) denotes strong (resp. weak) convergence.

Lemma $2.2([8])$. Let $C$ be a nonempty closed convex subset of a Hilbert space $H$ and suppose that $T: C \rightarrow H$ is nonexpansive. Then, the mapping $I-T$ is demiclosed at zero.

Lemma $2.3([11])$. Let $(E,\langle\cdot, \cdot\rangle)$ be an inner product space. Then for all $x, y, z \in E$ and $\alpha, \beta, \gamma \in[0,1]$ with $\alpha+\beta+\gamma=1$, we have

$$
\begin{gathered}
\|\alpha x+\beta y+\gamma z\|^{2}=\alpha\|x\|^{2}+\beta\|y\|^{2} \gamma\|z\|^{2}-\alpha \beta\|x-y\|^{2} \\
-\alpha \gamma\|x-z\|^{2}-\beta \gamma\|y-z\|^{2} .
\end{gathered}
$$

Lemma 2.4 ([1]). Let $H$ be a real Hilbert space. Then, for all $x, y \in H$

(i) $\|x-y\|^{2} \leq\|x\|^{2}+2\langle y, x+y\rangle$,

(ii) $\|x-y\|^{2} \geq\|x\|^{2}+2\langle y, x\rangle$.

Lemma 2.5 ([13]). Let $C$ be a nonempty closed convex subset of a real Hilbert space $H$.

(i) If $F: C \rightarrow C$ is a mapping which is both $\delta$-strongly monotone and $\lambda$-strictly pseudo-contractive of Browder-Petryshyn type such that $\delta>$ $\frac{1+\lambda}{2}$. Then, $I-F$ is contractive with constant $\sqrt{\frac{2-2 \delta}{1-\lambda}}$.

(ii) If $F: C \rightarrow C$ is a mapping which is both $\delta$-strongly monotone and $\lambda$-strictly pseudo-contractive of Browder-Petryshyn type such that $\delta>$ $\frac{1+\lambda}{2}$. Then, for any fixed number $\tau \in(0,1), I-\tau F$ is contractive with constant $1-\tau\left(1-\sqrt{\frac{2-2 \delta}{1-\lambda}}\right)$. 
Lemma 2.6 ([20]). Let $\left\{a_{n}\right\}$ be a sequence of nonnegative real numbers such that

$$
a_{n+1} \leq\left(1-b_{n}\right) a_{n}+b_{n} c_{n}, \quad n \geq 0
$$

where $\left\{b_{n}\right\}$ and $\left\{c_{n}\right\}$ are sequences of real numbers satisfying the following conditions:

(i) $\left\{b_{n}\right\} \subset(0,1), \sum_{n=0}^{\infty} b_{n}=\infty$,

(ii) either $\lim \sup _{n \rightarrow \infty} c_{n} \leq 0$ or $\sum_{n=0}^{\infty}\left|b_{n} c_{n}\right|<\infty$.

Then, $\lim _{n \rightarrow \infty} a_{n}=0$.

\section{Strong convergence theorems}

The following is our main result.

Theorem 3.1. Let $C$ be a nonempty closed convex subset of a real Hilbert space $H$. Let $F: C \rightarrow C$ be a mapping which is both $\delta$-strongly monotone and $\lambda$-strictly pseudo-contractive of Browder-Petryshyn type such that $\delta>(1+\lambda) / 2$, $f: C \rightarrow C$ a contraction with coefficient $0<\alpha<1$ and $\gamma$ be a positive real number such that $\gamma<\left(1-\sqrt{\frac{2-2 \delta}{1-\lambda}}\right) / \alpha$. Let $T: C \rightarrow C$ be a nonexpansive mapping and for each $i=1,2, \ldots, m+2$, let $A_{i}: C \rightarrow H$ be a $\delta_{i}$-inverse strongly monotone mapping and $\mathcal{F}=\bigcap_{i=1}^{m+2} V I\left(C, A_{i}\right) \cap F i x(T) \neq \emptyset$. Let $\left\{\alpha_{n}\right\}_{n=1}^{\infty},\left\{\gamma_{i, n}\right\}_{i=1, n=1}^{m, \infty}$ and $\left\{\delta_{i, n}\right\}_{i=1, n=1}^{m, \infty}$ be sequences in $(0,1)$, and $\left\{\beta_{n}\right\}_{n=1}^{\infty}$ be a sequence in $[0,1)$ satisfy the following conditions:

$\left(B_{1}\right) \gamma_{i, n}+\gamma_{i+1, n}+\gamma_{i+2, n}=1,0<\liminf _{n \rightarrow \infty} \gamma_{i, n} \leq \limsup _{n \rightarrow \infty} \gamma_{i, n}<1$ and $\left\{\delta_{i, n}\right\} \subset\left(0,2 \delta_{i}\right), \quad i=1,2, \ldots, m$

$\left(B_{2}\right) \lim _{n \rightarrow \infty} \alpha_{n}=0, \sum_{n=1}^{\infty} \alpha_{n}=\infty$ and $\lim _{n \rightarrow \infty} \beta_{n}=0$.

$\left(B_{3}\right) \sum_{n=1}^{\infty}\left|\alpha_{n+1}-\alpha_{n}\right|<\infty, \sum_{n=1}^{\infty}\left|\beta_{n+1}-\beta_{n}\right|<\infty, \sum_{n=1}^{\infty}\left|\delta_{i, n+1}-\delta_{i, n}\right|$ $<\infty, \sum_{n=1}^{\infty}\left|\gamma_{i, n+1}-\gamma_{i, n}\right|<\infty, \quad i=1,2, \ldots, m+2$.

Suppose $\left\{x_{n}\right\}_{n=1}^{\infty}$ and $\left\{y_{n}\right\}_{n=1}^{\infty}$ are sequences generated by $x_{0} \in C$ and

$$
\begin{gathered}
x_{n+1}=\alpha_{n} \gamma f\left(T P_{C}\left(I-\delta_{1, n} A_{1}\right) y_{1, n}\right)+\beta_{n} P_{C}\left(I-\delta_{2, n} A_{2}\right) x_{n} \\
+\left(\left(1-\beta_{n}\right) I-\alpha_{n} F\right) T P_{C}\left(I-\delta_{3, n} A_{3}\right) y_{1, n}, \\
y_{i, n}=\gamma_{i, n} P_{C}\left(I-\delta_{i, n} A_{i}\right) y_{i+1, n}+\gamma_{i+1, n} P_{C}\left(I-\delta_{i+1, n} A_{i+1}\right) y_{i+1, n} \\
+\gamma_{i+2, n} P_{C}\left(I-\delta_{i+2, n} A_{i+2}\right) y_{i+1, n}, \quad i=1,2, \ldots, m,
\end{gathered}
$$

(8) $y_{m+1, n}=x_{n}$.

Then $\left\{x_{n}\right\}_{n=1}^{\infty}$ and $\left\{y_{i, n}\right\}_{i=1, n=1}^{m, \infty}$ converge strongly to $x^{*} \in \mathcal{F}$, which is the unique solution of the system of variational inequalities:

$$
\left\{\begin{array}{l}
\left\langle(F-\gamma f) x^{*}, x-x^{*}\right\rangle \geq 0 \\
\left\langle A_{i} x^{*}, x-x^{*}\right\rangle \geq 0, \quad \forall x \in \mathcal{F}, i=1,2, \ldots, m+2 .
\end{array}\right.
$$

Proof. By taking

$$
\begin{aligned}
& u_{i, n}=P_{C}\left(I-\delta_{i, n} A_{i}\right) y_{i+1, n}, \\
& v_{i, n}=P_{C}\left(I-\delta_{i+1, n} A_{i+1}\right) y_{i+1, n},
\end{aligned}
$$




$$
w_{i, n}=P_{C}\left(I-\delta_{i+2, n} A_{i+2}\right) y_{i+1, n}, \quad i=1,2, \ldots, m .
$$

We shall equivalently write scheme (8) as follows:

$$
\begin{gathered}
x_{n+1}=\alpha_{n} \gamma f\left(T P_{C}\left(I-\delta_{1, n} A_{1}\right) y_{1, n}\right)+\beta_{n} P_{C}\left(I-\delta_{2, n} A_{2}\right) x_{n} \\
+\left(\left(1-\beta_{n}\right) I-\alpha_{n} F\right) T P_{C}\left(I-\beta_{3, n} A_{3}\right) y_{1, n}, \\
y_{i, n}=\gamma_{i, n} u_{i, n}+\gamma_{i+1, n} v_{i, n}+\gamma_{i+2, n} w_{i, n}, \quad i=1,2, \ldots, m
\end{gathered}
$$

Since $\left\{\delta_{i, n}\right\}_{i=1, n=1}^{m, \infty}$ satisfy in condition $\left(B_{1}\right)$ and $A_{i}$ is a $\delta_{i}$-inverse strongly monotone mapping, for any $x, y \in C$, we have

$$
\begin{aligned}
& \left\|\left(I-\delta_{i, n} A_{i}\right) x-\left(I-\delta_{i, n} A_{i}\right) y\right\|^{2} \\
= & \left\|(x-y)-\delta_{i, n}\left(A_{i} x-A_{i} y\right)\right\|^{2} \\
= & \|x-y\|^{2}-2 \delta_{i, n}\left\langle x-y, A_{i} x-A_{i} y\right\rangle+\delta_{i, n}^{2}\left\|A_{i} x-A_{i} y\right\|^{2} \\
\leq & \|x-y\|^{2}-2 \delta_{i, n} \delta_{i}\left\|A_{i} x-A_{i} y\right\|^{2}+\delta_{i, n}^{2}\left\|A_{i} x-A_{i} y\right\|^{2} \\
= & \|x-y\|^{2}+\delta_{i, n}\left(\delta_{i, n}-2 \delta_{i}\right)\left\|A_{i} x-A_{i} y\right\|^{2} \\
\leq & \|x-y\|^{2} .
\end{aligned}
$$

It follows that

$$
\left\|\left(I-\delta_{i, n} A_{i}\right) x-\left(I-\delta_{i, n} A_{i}\right) y\right\| \leq\|x-y\|, \quad i=1,2, \ldots, m+2 .
$$

Let $p \in \mathcal{F}$, in the context of the variational inequality problem the characterization of projection (6) implies that $p=P_{C}\left(I-\beta_{i, n} A_{i}\right) p, \quad i=1,2, \ldots, m+2$. Using condition $\left(B_{1}\right),(10)$ and Lemma 2.3, we get

$$
\begin{aligned}
& \left\|y_{i, n}-p\right\|^{2} \\
= & \| \gamma_{i, n} P_{C}\left(I-\delta_{i, n} A_{i}\right) y_{i+1, n}+\gamma_{i+1, n} P_{C}\left(I-\delta_{i+1, n} A_{i+1}\right) y_{i+1, n} \\
& +\gamma_{i+2, n} P_{C}\left(I-\delta_{i+2, n} A_{i+2}\right) y_{i+1, n}-p \|^{2} \\
\leq & \gamma_{i, n}\left\|P_{C}\left(I-\delta_{i, n} A_{i}\right) y_{i+1, n}-p\right\|^{2}+\gamma_{i+1, n}\left\|P_{C}\left(I-\delta_{i+1, n} A_{i+1}\right) y_{i+1, n}-p\right\|^{2} \\
& \quad+\gamma_{i+2, n}\left\|P_{C}\left(I-\delta_{i+2, n} A_{i+2}\right) y_{i+1, n}-p\right\|^{2} \\
\leq & \gamma_{i, n}\left\|y_{i+1, n}-p\right\|^{2}+\gamma_{i+1, n}\left\|y_{i+1, n}-p\right\|^{2}+\gamma_{i+2, n}\left\|y_{i+1, n}-p\right\|^{2} \\
= & \left(\gamma_{i, n}+\gamma_{i+1, n}+\gamma_{i+2, n}\right)\left\|y_{i+1, n}-p\right\|^{2}=\left\|y_{i+1, n}-p\right\|^{2} .
\end{aligned}
$$

Which implies that

(11) $\quad\left\|y_{1, n}-p\right\| \leq\left\|y_{2, n}-p\right\| \leq \cdots \leq\left\|y_{m, n}-p\right\| \leq\left\|y_{m+1, n}-p\right\|=\left\|x_{n}-p\right\|$.

First we show that $\left\{x_{n}\right\}$ is bounded. Indeed, we take $p \in \mathcal{F}$. Then using (6), (11) and Lemma 2.4, we have

$$
\begin{aligned}
& \left\|x_{n+1}-p\right\| \\
=\| & \alpha_{n} \gamma f\left(T P_{C}\left(I-\delta_{1, n} A_{1} y_{1, n}\right)+\beta_{n} P_{C}\left(I-\delta_{2, n} A_{2}\right) x_{n}\right. \\
& +\left(\left(1-\beta_{n}\right) I-\alpha_{n} F\right) T P_{C}\left(I-\delta_{3, n} A_{3}\right) y_{1, n}-p \|
\end{aligned}
$$




$$
\begin{aligned}
= & \|\left[\left(\left(1-\beta_{n}\right) I-\alpha_{n} F\right) T P_{C}\left(I-\delta_{3, n} A_{3}\right) y_{1, n}-\left(\left(1-\beta_{n}\right) I-\alpha_{n} F\right) p\right] \\
& +\beta_{n}\left[P_{C}\left(I-\delta_{2, n} A_{2}\right) x_{n}-p\right]+\alpha_{n}\left[\gamma f\left(T P_{C}\left(I-\delta_{1, n} A_{1}\right) y_{1, n}-F(p)\right] \|\right. \\
\leq & \left(1-\beta_{n}-\alpha_{n}\left(1-\sqrt{\frac{2-2 \delta}{1-\lambda}}\right)\right)\left\|y_{1, n}-p\right\|+\beta_{n}\left\|x_{n}-p\right\| \\
& +\alpha_{n}\left\|\gamma f\left(T P_{C}\left(I-\delta_{1, n} A_{1}\right) y_{1, n}\right)-\gamma f(p)\right\|+\alpha_{n}\|\gamma f(p)-F(p)\| \\
\leq & \left(1-\beta_{n}-\alpha_{n}\left(1-\sqrt{\frac{2-2 \delta}{1-\lambda}}-\gamma \alpha\right)\right)\left\|y_{1, n}-p\right\| \\
& +\beta_{n}\left\|x_{n}-p\right\|+\alpha_{n}\|\gamma f(p)-F(p)\| \\
\leq & \left(1-\alpha_{n}\left(1-\sqrt{\frac{2-2 \delta}{1-\lambda}}-\gamma \alpha\right)\right)\left\|x_{n}-p\right\| \\
& +\frac{\alpha_{n}\left(1-\sqrt{\frac{2-2 \delta}{1-\lambda}}-\gamma \alpha\right)}{\left(1-\sqrt{\frac{2-2 \delta}{1-\lambda}}-\gamma \alpha\right)}\|\gamma f(p)-F(p)\| \\
\leq & \max \left\{\left(1-\sqrt{\frac{2-2 \delta}{1-\lambda}}-\gamma \alpha\right)^{-1}\|\gamma f(p)-F(p)\|,\left\|x_{n}-p\right\|\right\} .
\end{aligned}
$$

By induction,

$$
\left\|x_{n}-p\right\| \leq \max \left\{\left(1-\sqrt{\frac{2-2 \delta}{1-\lambda}}-\gamma \alpha\right)^{-1}\|\gamma f(p)-F(p)\|,\left\|x_{0}-p\right\|\right\} .
$$

Therefore, $\left\{x_{n}\right\}$ is bounded and so are the sequences $\left\{y_{i, n}\right\}_{i=1, n=1}^{m, \infty}$. Now we claim that

$$
\lim _{n \rightarrow \infty}\left\|x_{n+1}-x_{n}\right\|=0 .
$$

From the definition of $\left\{y_{i, n}\right\}$, we have

$$
\begin{aligned}
& \quad\left\|y_{i, n+1}-y_{i, n}\right\| \\
& =\| \gamma_{i, n+1} u_{i, n+1}+\gamma_{i+1, n+1} v_{i, n+1}+\gamma_{i+2, n+1} w_{i, n+1}-\gamma_{i, n+1} u_{i, n} \\
& \quad-\gamma_{i+1, n} v_{i, n}-\gamma_{i+2, n} w_{i, n} \| \\
& =\| \gamma_{i, n+1}\left(u_{i, n+1}-u_{i, n}\right)+\left(\gamma_{i, n+1}-\gamma_{i, n}\right) u_{i, n}+\gamma_{i+1, n+1}\left(v_{i, n+1}-v_{i, n}\right) \\
& \quad+\left(\gamma_{i+1, n+1}-\gamma_{i+1, n}\right) v_{i, n}+\gamma_{i+2, n+1}\left(w_{i, n+1}-w_{i, n}\right) \\
& \quad+\left(\gamma_{i+2, n+1}-\gamma_{i+2, n}\right) w_{i, n} \| \\
& \leq \gamma_{i, n+1}\left\|u_{i, n+1}-u_{i, n}\right\|+\gamma_{i+1, n+1}\left\|v_{i, n+1}-v_{i, n}\right\| \\
& \quad+\gamma_{i+2, n+1}\left\|w_{i, n+1}-w_{i, n}\right\|+\left|\gamma_{i, n+1}-\gamma_{i, n}\right|\left\|u_{i, n}\right\| \\
& \quad+\left|\gamma_{i+1, n+1}-\gamma_{i+1, n}\right|\left\|v_{i, n}\right\|+\left|\gamma_{i+2, n+1}-\gamma_{i+2, n}\right|\left\|w_{i, n}\right\| \\
& =\gamma_{i, n+1} \| P_{C}\left(I-\delta_{i, n+1} A_{i}\right) y_{i+1, n+1}-P_{C}\left(I-\delta_{i, n+1} A_{i}\right) y_{i+1, n}
\end{aligned}
$$




$$
\begin{aligned}
& +P_{C}\left(I-\delta_{i, n+1} A_{i}\right) y_{i+1, n}-P_{C}\left(I-\delta_{i, n+1} A_{i}\right) y_{i+1, n} \| \\
& +\gamma_{i+1, n+1} \| P_{C}\left(I-\delta_{i+1, n+1} A_{i+1}\right) y_{i+1, n+1}-P_{C}\left(I-\delta_{i+1, n+1} A_{i+1}\right) y_{i+1, n} \\
& +P_{C}\left(I-\delta_{i+1, n+1} A_{i+1}\right) y_{i+1, n}-P_{C}\left(I-\delta_{i+1, n} A_{i+1}\right) y_{i+1, n} \| \\
& +\gamma_{i+2, n+1} \| P_{C}\left(I-\delta_{i+2, n+1} A_{i+2}\right) y_{i+1, n+1}-P_{C}\left(I-\delta_{i+2, n+1} A_{i+2}\right) y_{i+1, n} \\
& +P_{C}\left(I-\delta_{i+2, n+1} A_{i+2}\right) y_{i+1, n}-P_{C}\left(I-\delta_{i+2, n} A_{i+2}\right) y_{i+1, n} \| \\
& +\left|\gamma_{i, n+1}-\gamma_{i, n}\right|\left\|u_{i, n}\right\|+\left|\gamma_{i+1, n+1}-\gamma_{i+1, n}\right|\left\|v_{i, n}\right\| \\
& +\left|\gamma_{i+2, n+1}-\gamma_{i+2, n}\right|\left\|w_{i, n}\right\| \\
\leq & \gamma_{i, n+1}\left\|y_{i+1, n+1}-y_{i+1, n}\right\| \\
& +\gamma_{i, n+1}\left|\delta_{i, n+1}-\delta_{i, n+1}\right|\left\|A_{i} y_{i+1, n}\right\| \\
& +\gamma_{i+1, n+1}\left\|y_{i+1, n+1}-y_{i+1, n}\right\| \\
& +\gamma_{i+1, n+1}\left|\delta_{i+1, n+1}-\delta_{i+1, n}\right|\left\|A_{i+1} y_{i+1, n}\right\| \\
& +\gamma_{i+2, n+1}\left\|y_{i+1, n+1}-y_{i+1, n}\right\| \\
& +\gamma_{i+2, n+1}\left|\delta_{i+2, n+1}-\delta_{i+2, n}\right|\left\|A_{i+2} y_{i+1, n}\right\| \\
& +\left|\gamma_{i, n+1}-\gamma_{i, n}\right|\left\|u_{i, n}\right\|+\left|\gamma_{i+1, n+1}-\gamma_{i+1, n}\right|\left\|v_{i, n}\right\| \\
& +\left|\gamma_{i+2, n+1}-\gamma_{i+2, n}\right|\left\|w_{i, n}\right\| \\
= & \gamma_{i, n+1}\left\|y_{i+1, n+1}-y_{i+1, n}\right\|+\gamma_{i, n+1}\left|\delta_{i, n+1}-\delta_{i, n+1}\right|\left\|A_{i} y_{i+1, n}\right\| \\
& +\gamma_{i+1, n+1} \mid \delta_{i+1, n+1}-\delta_{i+1, n}\left\|A_{i+1} y_{i+1, n}\right\| \\
& +\gamma_{i+2, n+1}\left|\delta_{i+2, n+1}-\delta_{i+2, n}\right|\left\|A_{i+2} y_{i+1, n}\right\| \\
& +\left|\gamma_{i, n+1}-\gamma_{i, n}\right|\left\|u_{i, n}\right\|+\left|\gamma_{i+1, n+1}-\gamma_{i+1, n}\right|\left\|v_{i, n}\right\| \\
& +\left|\gamma_{i+2, n+1}-\gamma_{i+2, n}\right|\left\|w_{i, n}\right\|
\end{aligned}
$$

which implies that for some approximate constant $M_{i}>0$,

$$
\begin{aligned}
& \left\|y_{i, n+1}-y_{i, n}\right\| \\
\leq & \left\|y_{i+1, n+1}-y_{i+1, n}\right\|+\left[\left|\delta_{i, n+1}-\delta_{i, n}\right|+\left|\delta_{i+1, n+1}-\delta_{i+1, n}\right|\right. \\
& +\left|\delta_{i+2, n+1}-\delta_{i+2, n}\right|+\left|\gamma_{i, n+1}-\gamma_{i, n}\right|+\left|\gamma_{i, n+1}-\gamma_{i, n}\right| \\
& \left.+\left|\gamma_{i+2, n+1}-\gamma_{i+2, n}\right|\right] M_{i} .
\end{aligned}
$$

It follows that

$$
\begin{aligned}
& \left\|y_{i, n+1}-y_{i, n}\right\| \\
\leq & \left\|x_{n+1}-x_{n}\right\|+\sum_{j=i}^{m}\left[\left|\delta_{j, n+1}-\delta_{j, n}\right|+\left|\delta_{j+1, n+1}-\delta_{j+1, n}\right|\right. \\
& +\left|\delta_{j+2, n+1}-\delta_{i+2, n}\right|+\left|\gamma_{j, n+1}-\gamma_{j, n}\right|+\left|\gamma_{j, n+1}-\gamma_{j, n}\right| \\
& \left.+\left|\gamma_{j+2, n+1}-\gamma_{j+2, n}\right|\right] M_{j} .
\end{aligned}
$$

Indeed, we have

$$
\left\|x_{n+1}-x_{n}\right\|
$$




$$
\begin{aligned}
& =\| \alpha_{n} \gamma f\left(T P_{C}\left(I-\delta_{1, n} A_{1}\right) y_{1, n}\right)+\beta_{n} P_{C}\left(I-\delta_{2, n} A_{2}\right) x_{n} \\
& +\left(\left(1-\beta_{n}\right) I-\alpha_{n} F\right) T P_{C}\left(I-\delta_{3, n} A_{3}\right) y_{1, n} \\
& -\alpha_{n-1} \gamma f\left(T P_{C}\left(I-\delta_{1, n-1} A_{1}\right) y_{1, n-1}\right)-\beta_{n-1} P_{C}\left(I-\delta_{2, n-1} A_{2}\right) x_{n-1} \\
& -\left(\left(1-\beta_{n-1}\right) I-\alpha_{n-1} F\right) T P_{C}\left(I-\delta_{3, n-1} A_{3}\right) y_{1, n-1} \| \\
& =\|\left[\left(\left(1-\beta_{n}\right) I-\alpha_{n} F\right) T P_{C}\left(I-\delta_{3, n} A_{3}\right) y_{1, n}\right. \\
& \left.-\left(\left(1-\beta_{n}\right) I-\alpha_{n} F\right) T P_{C}\left(I-\delta_{3, n-1} A_{3}\right) y_{1, n-1}\right] \\
& +\left[\left(\left(1-\beta_{n}\right) I-\alpha_{n} F\right) T P_{C}\left(I-\delta_{3, n-1} A_{3}\right) y_{1, n-1}\right. \\
& \left.-\left(\left(1-\beta_{n-1}\right) I-\alpha_{n-1} F\right) T P_{C}\left(I-\delta_{3, n-1} A_{3}\right) y_{1, n-1}\right] \\
& +\beta_{n}\left[P_{C}\left(I-\delta_{2, n} A_{2}\right) x_{n}-P_{C}\left(I-\delta_{2, n} A_{2}\right) x_{n-1}\right] \\
& +\beta_{n}\left[P_{C}\left(I-\delta_{2, n} A_{2}\right) x_{n-1}-P_{C}\left(I-\delta_{2, n-1} A_{2}\right) x_{n-1}\right] \\
& +\left(\beta_{n}-\beta_{n-1}\right) P_{C}\left(I-\delta_{2, n-1} A_{2}\right) x_{n-1}+\alpha_{n} \gamma\left[f\left(T P_{C}\left(I-\delta_{1, n} A_{1}\right) y_{1, n}\right)\right. \\
& \left.-f\left(T P_{C}\left(I-\delta_{1, n-1} A_{1}\right) y_{1, n-1}\right)\right] \\
& +\left(\alpha_{n}-\alpha_{n-1}\right) \gamma f\left(T P_{C}\left(I-\delta_{1, n-1} A_{1}\right) y_{1, n-1}\right) \| \\
& \leq\left(1-\beta_{n}-\alpha_{n}\left(1-\sqrt{\frac{2-2 \delta}{1-\lambda}}\right)\right)\left\|\left(I-\delta_{3, n} A_{3}\right) y_{1, n}-\left(I-\delta_{3, n-1} A_{3}\right) y_{1, n-1}\right\| \\
& +\left|\beta_{n}-\beta_{n-1}\right||| T P_{C}\left(I-\delta_{3, n-1} A_{3}\right) y_{1, n-1} \| \\
& +\left|\alpha_{n}-\alpha_{n-1}\right||| F T P_{C}\left(I-\beta_{3, n-1} A_{3}\right) y_{1, n-1} \| \\
& +\beta_{n}\left\|x_{n}-x_{n-1}\right\|+\beta_{n}\left|\delta_{2, n}-\delta_{2, n-1}\right|\left\|A_{2} x_{n-1}\right\| \\
& +\left|\beta_{n}-\beta_{n-1}\right||| P_{C}\left(I-\delta_{2, n-1} A_{2}\right) x_{n-1}\left\|+\alpha_{n} \gamma \alpha\right\| y_{1, n}-y_{1, n-1} \| \\
& +\left|\alpha_{n}-\alpha_{n-1}\right| \gamma\left\|f\left(T P_{C}\left(I-\delta_{1, n-1} A_{1}\right) y_{1, n-1}\right)\right\| \\
& \leq\left(1-\beta_{n}-\alpha_{n}\left(1-\sqrt{\frac{2-2 \delta}{1-\lambda}}-\gamma \alpha\right)\right)\left\|y_{1, n}-y_{1, n-1}\right\| \\
& +\left(1-\beta_{n}-\alpha_{n}\left(1-\sqrt{\frac{2-2 \delta}{1-\lambda}}-\right)\right)\left|\delta_{3, n}-\delta_{3, n-1}\right|\left\|A_{3} y_{1, n-1}\right\| \\
& +\left|\beta_{n}-\beta_{n-1}\right||| T P_{C}\left(I-\delta_{3, n-1} A_{3}\right) y_{n-1}|| \\
& +\left|\alpha_{n}-\alpha_{n-1}\right||| F T P_{C}\left(I-\delta_{3, n-1} A_{3}\right) y_{1, n-1} \| \\
& +\beta_{n}\left\|x_{n}-x_{n-1}\right\|+\beta_{n}\left|\delta_{2, n}-\delta_{2, n-1}\right|\left\|A_{2} x_{n-1}\right\| \\
& +\left|\beta_{n}-\beta_{n-1}\right||| P_{C}\left(I-\delta_{2, n-1} A_{2}\right) x_{n-1} \| \\
& +\left|\alpha_{n}-\alpha_{n-1}\right| \gamma\left\|f\left(T P_{C}\left(I-\delta_{1, n-1} A_{1}\right) y_{1, n-1}\right)\right\| \text {, }
\end{aligned}
$$

which implies that for some approximate constant $M>0$,

(14) $\quad\left\|x_{n+1}-x_{n}\right\|$

$$
\leq\left(1-\beta_{n}-\alpha_{n}\left(1-\sqrt{\frac{2-2 \delta}{1-\lambda}}-\gamma \alpha\right)\right)\left\|y_{1, n}-y_{1, n-1}\right\|
$$




$$
\begin{aligned}
& +\beta_{n}\left\|x_{n}-x_{n-1}\right\|+\left[\left|\delta_{3, n}-\delta_{3, n-1}\right|+\left|\beta_{n}-\beta_{n-1}\right|+\left|\alpha_{n}-\alpha_{n-1}\right|\right. \\
& \left.+\left|\delta_{2, n}-\delta_{2, n-1}\right|+\left|\beta_{n}-\beta_{n-1}\right|\left|\alpha_{n}-\alpha_{n-1}\right|\right] M .
\end{aligned}
$$

Substituting (13) into (14), we obtain

$$
\begin{aligned}
& \left\|x_{n+1}-x_{n}\right\| \\
\leq & \left(1-\alpha_{n}\left(1-\sqrt{\frac{2-2 \delta}{1-\lambda}}-\gamma \alpha\right)\right)\left\|x_{n}-x_{n-1}\right\| \\
& +\sum_{j=1}^{m}\left[\left|\delta_{j, n}-\delta_{j, n-1}\right|+\left|\delta_{j+1, n}-\delta_{j+1, n-1}\right|+\left|\gamma_{j, n}-\gamma_{j, n-1}\right|\right] M_{j} \\
& +\left[\left|\delta_{3, n}-\delta_{3, n-1}\right|+\left|\beta_{n}-\beta_{n-1}\right|+\left|\alpha_{n}-\alpha_{n-1}\right|\right. \\
& \left.+\left|\delta_{2, n}-\delta_{2, n-1}\right|+\left|\beta_{n}-\beta_{n-1}\right|\left|\alpha_{n}-\alpha_{n-1}\right|\right] M .
\end{aligned}
$$

Thus, using condition $\left(B_{3}\right)$ and Lemma 2.6 to (15), we conclude that

$$
\lim _{n \rightarrow \infty}\left\|x_{n+1}-x_{n}\right\|=0 .
$$

In this stage we well show that

$$
\begin{aligned}
& \lim _{n \rightarrow \infty}\left\|u_{i, n}-y_{i+1, n}\right\|=0, \\
& \lim _{n \rightarrow \infty}\left\|v_{i, n}-y_{i+1, n}\right\|=0, \\
& \lim _{n \rightarrow \infty}\left\|w_{i, n}-y_{i+1, n}\right\|=0, \quad i=1,2, \ldots, m .
\end{aligned}
$$

By $p \in \mathcal{F}$ in (11) and the definition of $\left\{x_{n}\right\}$, we have

$$
\begin{aligned}
& \left\|x_{n+1}-p\right\|^{2} \\
= & \| \alpha_{n} \gamma f\left(T P_{C}\left(I-\delta_{1, n} A_{1}\right) y_{1, n}\right)+\beta_{n} P_{C}\left(I-\delta_{2, n} A_{2}\right) x_{n} \\
& +\left(\left(1-\beta_{n}\right) I-\alpha_{n} F\right) T P_{C}\left(I-\delta_{3, n} A_{3}\right) y_{1, n}-p \|^{2} \\
= & \|\left[\left(\left(1-\beta_{n}\right) I-\alpha_{n} F\right) T P_{C}\left(I-\delta_{3, n} A_{3}\right) y_{1, n}-\left(\left(1-\beta_{n}\right) I-\alpha_{n} F\right) p\right] \\
& +\beta_{n}\left[P_{C}\left(I-\delta_{2, n} A_{2}\right) x_{n}-p\right]+\alpha_{n}\left[\gamma f\left(T P_{C}\left(I-\delta_{1, n} A_{1}\right) y_{1, n}\right)-F p\right] \|^{2} \\
\leq & \left\|\left(\left(1-\beta_{n}\right) I-\alpha_{n} F\right) T P_{C}\left(I-\delta_{3, n} A_{3}\right) y_{1, n}-\left(\left(1-\beta_{n}\right) I-\alpha_{n} F\right) p\right\|^{2} \\
& +2 \beta_{n}\left\langle P_{C}\left(I-\delta_{2, n} A_{2}\right) x_{n}-p, x_{n+1}-p\right\rangle \\
+ & 2 \alpha_{n}\left\langle\gamma f\left(T P_{C}\left(I-\delta_{1, n} A_{1}\right) y_{1, n}\right)-F p, x_{n+1}-p\right\rangle \\
& \leq\left(1-\beta_{n}-\alpha_{n}\left(1-\sqrt{\frac{2-2 \delta}{1-\lambda}}-\gamma \alpha\right)\right)\left\|y_{1, n}-p\right\|^{2} \\
& +2 \beta_{n}\left\langle P_{C}\left(I-\delta_{2, n} A_{2}\right) x_{n}-p, x_{n+1}-p\right\rangle \\
+ & 2 \alpha_{n}\left\langle\gamma f\left(T P_{C}\left(I-\delta_{1, n} A_{1}\right) y_{1, n}\right)-F p, x_{n+1}-p\right\rangle \\
\leq & \left\|y_{1, n}-p\right\|^{2}+2 \beta_{n}\left\langle P_{C}\left(I-\delta_{2, n} A_{2}\right) x_{n}-p, x_{n+1}-p\right\rangle \\
+ & 2 \alpha_{n}\left\langle\gamma f\left(T P_{C}\left(I-\delta_{1, n} A_{1}\right) y_{1, n}\right)-F p, x_{n+1}-p\right\rangle
\end{aligned}
$$




$$
\begin{aligned}
\leq & \left\|y_{i, n}-p\right\|^{2}+2 \beta_{n}\left\langle P_{C}\left(I-\delta_{2, n} A_{2}\right) x_{n}-p, x_{n+1}-p\right\rangle \\
& +2 \alpha_{n}\left\langle\gamma f\left(T P_{C}\left(I-\delta_{1, n} A_{1}\right) y_{1, n}\right)-F p, x_{n+1}-p\right\rangle .
\end{aligned}
$$

From (6), (11), (17) and Lemma 2.3, we have (for $i=1,2, \ldots, m$ )

$$
\begin{aligned}
& \left\|x_{n+1}-p\right\|^{2} \\
\leq & \| \gamma_{i, n} P_{C}\left(I-\delta_{i, n} A_{i}\right) y_{i+1, n}+\gamma_{i+1, n} P_{C}\left(I-\delta_{i+1, n} A_{i+1}\right) y_{i+1, n} \\
& +\gamma_{i+2, n} P_{C}\left(I-\delta_{i+2, n} A_{i+2}\right) y_{i+1, n}-p \|^{2} \\
& +2 \beta_{n}\left\langle P_{C}\left(I-\delta_{2, n} A_{2}\right) x_{n}-p, x_{n+1}-p\right\rangle \\
& +2 \alpha_{n}\left\langle\gamma f\left(T P_{C}\left(I-\delta_{1, n} A_{1}\right) y_{1, n}\right)-F p, x_{n+1}-p\right\rangle \\
\leq & \gamma_{i, n}\left\|P_{C}\left(I-\delta_{i, n} A_{i}\right) y_{i+1, n}-p\right\|^{2}+\gamma_{i+1, n}\left\|P_{C}\left(I-\delta_{i+1, n} A_{i+1}\right) y_{i+1, n}-p\right\|^{2} \\
& +\gamma_{i+2, n}\left\|P_{C}\left(I-\delta_{i+2, n} A_{i+2}\right) y_{i+1, n}-p\right\|^{2} \\
& +2 \beta_{n}\left\langle P_{C}\left(I-\delta_{2, n} A_{2}\right) x_{n}-p, x_{n+1}-p\right\rangle \\
& +2 \alpha_{n}\left\langle\gamma f\left(T P_{C}\left(I-\delta_{1, n} A_{1}\right) y_{1, n}\right)-F p, x_{n+1}-p\right\rangle \\
\leq & \gamma_{i, n}\left\|\left(y_{i+1, n}-p\right)-\delta_{i, n}\left(A_{i} y_{i+1, n}-A_{i} p\right)\right\|^{2}+\gamma_{i+1, n}\left\|y_{i+1, n}-p\right\|^{2} \\
& +\gamma_{i+2, n}\left\|y_{i+1, n}-p\right\|^{2}+2 \beta_{n}\left\langle P_{C}\left(I-\delta_{2, n} A_{2}\right) x_{n}-p, x_{n+1}-p\right\rangle \\
& +2 \alpha_{n}\left\langle\gamma f\left(T P_{C}\left(I-\delta_{1, n} A_{1}\right) y_{1, n}\right)-F p, x_{n+1}-p\right\rangle \\
\leq & \gamma_{i, n}\left[\left\|y_{i+1, n}-p\right\|^{2}+\delta_{i, n}^{2}\left\|A_{i} y_{i+1, n}-A_{i} p\right\|^{2}\right. \\
& \left.-2 \delta_{i, n}\left\langle A_{i} y_{i+1, n}-A_{i} p, y_{i+1, n}-p\right\rangle\right]+\gamma_{i+1, n}\left\|y_{i+1, n}-p\right\|^{2} \\
& +\gamma_{i+2, n}\left\|y_{i+1, n}-p\right\|^{2}+2 \beta_{n}\left\langle P_{C}\left(I-\delta_{2, n} A_{2}\right) x_{n}-p, x_{n+1}-p\right\rangle \\
& +2 \alpha_{n}\left\langle\gamma f\left(T P_{C}\left(I-\delta_{1, n} A_{1}\right) y_{1, n}\right)-F p, x_{n+1}-p\right\rangle \\
\leq & \left(\gamma_{i, n}+\gamma_{i+1, n}+\gamma_{i+2, n}\right)\left\|y_{i+1, n}-p\right\|^{2}+\delta_{i, n}^{2}\left\|A_{i} y_{i+1, n}-A_{i} p\right\|^{2} \\
& -2 \delta_{i, n} \delta_{i}\left\|A_{i} y_{i+1, n}-A_{i} p\right\|^{2}+2 \beta_{n}\left\langle P_{C}\left(I-\delta_{2, n} A_{2}\right) x_{n}-p, x_{n+1}-p\right\rangle \\
& +2 \alpha_{n}\left\langle\gamma f\left(T P_{C}\left(I-\delta_{1, n} A_{1}\right) y_{1, n}\right)-F p, x_{n+1}-p\right\rangle \\
\leq & \left\|x_{n}-p\right\|^{2}+\delta_{i, n}\left(\delta_{i, n}-2 \delta_{i}\right)\left\|A_{i} y_{i+1, n}-A_{i} p\right\|^{2} \\
& +2 \beta_{n}\left\langle P_{C}\left(I-\delta_{2, n} A_{2}\right) x_{n}-p, x_{n+1}-p\right\rangle \\
& +2 \alpha_{n}\left\langle\gamma f\left(T P_{C}\left(I-\delta_{1, n} A_{1}\right) y_{1, n}\right)-F p, x_{n+1}-p\right\rangle \\
& +m
\end{aligned}
$$

which implies that

$$
\text { (18) } \begin{aligned}
& -\delta_{i, n}\left(\delta_{i, n}-2 \delta_{i}\right)\left\|A_{i} y_{i+1, n}-A_{i} p\right\|^{2} \\
\leq & \left\|x_{n}-p\right\|^{2}-\left\|x_{n+1}-p\right\|^{2}+2 \beta_{n}\left\langle P_{C}\left(I-\delta_{2, n} A_{2}\right) x_{n}-p, x_{n+1}-p\right\rangle \\
& +2 \alpha_{n}\left\langle\gamma f\left(T P_{C}\left(I-\delta_{1, n} A_{1}\right) y_{1, n}\right)-F p, x_{n+1}-p\right\rangle \\
\leq & {\left[\left\|x_{n}-p\right\|+\left\|x_{n+1}-p\right\|\right]\left\|x_{n+1}-x_{n}\right\| } \\
& +2 \beta_{n}\left\langle P_{C}\left(I-\delta_{2, n} A_{2}\right) x_{n}-p, x_{n+1}-p\right\rangle \\
& +2 \alpha_{n}\left\langle\gamma f\left(T P_{C}\left(I-\delta_{1, n} A_{1}\right) y_{1, n}\right)-F p, x_{n+1}-p\right\rangle .
\end{aligned}
$$


Using (12), (18) and condition $\left(B_{2}\right)$, we get

$$
\lim _{n \rightarrow \infty}\left\|A_{i} y_{i+1, n}-A_{i} p\right\|=0, \quad i=1,2, \ldots, m .
$$

Repeating the same argument as above, we conclude that

$$
\begin{aligned}
& \lim _{n \rightarrow \infty}\left\|A_{i+1} y_{i+1, n}-A_{i+1} p\right\|=0, \\
& \lim _{n \rightarrow \infty}\left\|A_{i+2} y_{i+1, n}-A_{i+2} p\right\|=0, i=1,2, \ldots, m .
\end{aligned}
$$

From (3), we have

$$
\begin{aligned}
& \left\|u_{i, n}-p\right\|^{2} \\
= & \left\|P_{C}\left(I-\delta_{i, n} A_{i}\right) y_{i+1, n}-P_{C}\left(I-\delta_{i, n} A_{i} p\right)\right\|^{2} \\
\leq & \left\langle\left(I-\delta_{i, n} A_{i}\right) y_{i+1, n}-\left(I-\delta_{i, n} A_{i}\right) p, u_{i, n}-p\right\rangle \\
= & \frac{1}{2}\left[\left\|\left(I-\delta_{i, n} A_{i}\right) y_{i+1, n}-\left(I-\delta_{i, n} A_{i}\right) p\right\|^{2}+\left\|u_{i, n}-p\right\|^{2}\right. \\
& \left.-\left\|\left(I-\delta_{i, n} A_{i}\right) y_{i+1, n}-\left(I-\delta_{i, n} A_{i}\right) p-\left(u_{i, n}-p\right)\right\|^{2}\right] \\
\leq & \frac{1}{2}\left[\left\|y_{i+1, n}-p\right\|^{2}+\left\|u_{i, n}-p\right\|^{2}\right. \\
& \left.-\left\|\left(I-\beta_{i, n} A_{i}\right) y_{i+1, n}-\left(I-\delta_{i, n} A_{i}\right) p-\left(u_{i, n}-p\right)\right\|^{2}\right] \\
= & \frac{1}{2}\left[\left\|y_{i+1, n}-p\right\|^{2}+\left\|u_{i, n}-p\right\|^{2}-\left\|y_{i+1, n}-u_{i, n}\right\|^{2}\right. \\
& \left.+2 \delta_{i, n}\left\langle y_{i+1, n}-u_{i, n}, A_{i} y_{i+1, n}-A_{i} p\right\rangle-\delta_{i, n}^{2}\left\|A_{i} y_{i+1, n}-A_{i} p\right\|^{2}\right] .
\end{aligned}
$$

So we obtain

$$
\begin{aligned}
\left\|u_{i, n}-p\right\|^{2} \leq & \left\|y_{i+1, n}-p\right\|^{2}-\left\|y_{i+1, n}-u_{i, n}\right\|^{2} \\
& +2 \delta_{i, n}\left\langle y_{i+1, n}-u_{i, n}, A_{i} y_{i+1, n}-A_{i} p\right\rangle \\
& -\delta_{i, n}^{2}\left\|A_{i} y_{i+1, n}-A_{i} p\right\|^{2}, \quad i=1,2, \ldots, m .
\end{aligned}
$$

By using some method as (21), we have

$$
\begin{aligned}
\left\|v_{i, n}-p\right\|^{2} \leq \| & y_{i+1, n}-p\left\|^{2}-\right\| y_{i+1, n}-v_{i, n} \|^{2} \\
& +2 \delta_{i+1, n}\left\langle y_{i+1, n}-v_{i, n}, A_{i+1} y_{i+1, n}-A_{i+1} p\right\rangle \\
& -\delta_{i+1, n}^{2}\left\|A_{i+1} y_{i+1, n}-A_{i+1} p\right\|^{2}, \quad i=1,2, \ldots, m
\end{aligned}
$$

and

$$
\begin{aligned}
\left\|w_{i, n}-p\right\|^{2} \leq \| & y_{i+1, n}-p\left\|^{2}-\right\| y_{i+1, n}-w_{i, n} \|^{2} \\
& +2 \delta_{i+2, n}\left\langle y_{i+1, n}-v_{i, n}, A_{i+2} y_{i+1, n}-A_{i+2} p\right\rangle \\
& -\delta_{i+2, n}^{2}\left\|A_{i+2} y_{i+1, n}-A_{i+2} p\right\|^{2}, \quad i=1,2, \ldots, m .
\end{aligned}
$$

From (17), (21), (22), (23) and Lemma 2.3, we have

$$
\begin{aligned}
& \left\|x_{n+1}-p\right\|^{2} \\
\leq & \left\|y_{i, n}-p\right\|^{2}+2 \beta_{n}\left\langle P_{C}\left(I-\delta_{2, n} A_{2}\right) x_{n}-p, x_{n+1}-p\right\rangle
\end{aligned}
$$




$$
\begin{aligned}
& +2 \alpha_{n}\left\langle\gamma f\left(T P_{C}\left(I-\delta_{1, n} A_{1}\right) y_{1, n}\right)-F p, x_{n+1}-p\right\rangle \\
& \leq \| \gamma_{i, n} P_{C}\left(I-\delta_{i, n} A_{i}\right) y_{i+1, n}+\gamma_{i+1, n} P_{C}\left(I-\delta_{i+1, n} A_{i+1}\right) y_{i+1, n} \\
& +\gamma_{i+2, n} P_{C}\left(I-\delta_{i+2, n} A_{i+2}\right) y_{i+1, n}-p \|^{2} \\
& +2 \beta_{n}\left\langle P_{C}\left(I-\delta_{2, n} A_{2}\right) x_{n}-p, x_{n+1}-p\right\rangle \\
& +2 \alpha_{n}\left\langle\gamma f\left(T P_{C}\left(I-\delta_{1, n} A_{1}\right) y_{1, n}\right)-F p, x_{n+1}-p\right\rangle \\
& \leq \gamma_{i, n}\left\|u_{i, n}-p\right\|^{2}+\gamma_{i+1, n}\left\|v_{i, n}-p\right\|^{2} \\
& +\gamma_{i+2, n}\left\|w_{i, n}-p\right\|^{2}+2 \beta_{n}\left\langle P_{C}\left(I-\delta_{2, n} A_{2}\right) x_{n}-p, x_{n+1}-p\right\rangle \\
& +2 \alpha_{n}\left\langle\gamma f\left(T P_{C}\left(I-\delta_{1, n} A_{1}\right) y_{1, n}\right)-F p, x_{n+1}-p\right\rangle \\
& \leq \gamma_{i, n}\left[\left\|y_{i+1, n}-p\right\|^{2}-\left\|y_{i+1, n}-u_{i, n}\right\|^{2}\right. \\
& +2 \delta_{i, n}\left\langle y_{i+1, n}-u_{i, n}, A_{i} y_{i+1, n}-A_{i} p\right\rangle \\
& \left.-\delta_{i, n}^{2}\left\|A_{i} y_{i+1, n}-A_{i} p\right\|^{2}\right]+\gamma_{i+1, n}\left[\left\|y_{i+1, n}-p\right\|^{2}-\left\|y_{i+1, n}-v_{i, n}\right\|^{2}\right. \\
& +2 \delta_{i+1, n}\left\langle y_{i+1, n}-v_{i, n}, A_{i+1} y_{i+1, n}-A_{i+1} p\right\rangle \\
& \left.-\delta_{i+1, n}^{2}\left\|A_{i+1} y_{i+1, n}-A_{i+1} p\right\|^{2}\right] \\
& +\gamma_{i+2, n}\left[\left\|y_{i+1, n}-p\right\|^{2}-\left\|y_{i+1, n}-w_{i, n}\right\|^{2}\right. \\
& +2 \delta_{i+2, n}\left\langle y_{i+1, n}-v_{i, n}, A_{i+2} y_{i+1, n}-A_{i+2} p\right\rangle \\
& \left.-\delta_{i+2, n}^{2}\left\|A_{i+2} y_{i+1, n}-A_{i+2} p\right\|^{2}\right] \\
& +2 \beta_{n}\left\langle P_{C}\left(I-\delta_{2, n} A_{2}\right) x_{n}-p, x_{n+1}-p\right\rangle \\
& +2 \alpha_{n}\left\langle\gamma f\left(T P_{C}\left(I-\delta_{1, n} A_{1}\right) y_{1, n}\right)-F p, x_{n+1}-p\right\rangle \\
& =\left(\gamma_{i, n}+\gamma_{i+1, n}+\gamma_{i+2, n}\right)\left\|y_{i+1, n}-p\right\|^{2}+\gamma_{i, n}\left[-\left\|y_{i+1, n}-u_{i, n}\right\|^{2}\right. \\
& \left.+2 \delta_{i, n}\left\langle y_{i+1, n}-u_{i, n}, A_{i} y_{i+1, n}-A_{i} p\right\rangle-\delta_{i, n}^{2}\left\|A_{i} y_{i+1, n}-A_{i} p\right\|^{2}\right] \\
& +\gamma_{i+1, n}\left[-\left\|y_{i+1, n}-v_{i, n}\right\|^{2}\right. \\
& +2 \delta_{i+1, n}\left\langle y_{i+1, n}-v_{i, n}, A_{i+1} y_{i+1, n}-A_{i+1} p\right\rangle \\
& \left.-\delta_{i+1, n}^{2}\left\|A_{i+1} y_{i+1, n}-A_{i+1} p\right\|^{2}\right] \\
& +\gamma_{i+2, n}\left[-\left\|y_{i+1, n}-w_{i, n}\right\|^{2}+2 \delta_{i+2, n}\left\langle y_{i+1, n}-v_{i, n}, A_{i+2} y_{i+1, n}-A_{i+2} p\right\rangle\right. \\
& \left.-\delta_{i+2, n}^{2}\left\|A_{i+2} y_{i+1, n}-A_{i+2} p\right\|^{2}\right] \\
& +2 \beta_{n}\left\langle P_{C}\left(I-\delta_{2, n} A_{2}\right) x_{n}-p, x_{n+1}-p\right\rangle \\
& +2 \alpha_{n}\left\langle\gamma f\left(T P_{C}\left(I-\delta_{1, n} A_{1}\right) y_{1, n}\right)-F p, x_{n+1}-p\right\rangle \\
& \leq\left\|x_{n}-p\right\|^{2}+\gamma_{i, n}\left[-\left\|y_{i+1, n}-u_{i, n}\right\|^{2}+2 \delta_{i, n}\left\langle y_{i+1, n}-u_{i, n}, A_{i} y_{i+1, n}-A_{i} p\right\rangle\right. \\
& \left.-\delta_{i, n}^{2}\left\|A_{i} y_{i+1, n}-A_{i} p\right\|^{2}\right]+\gamma_{i+1, n}\left[-\left\|y_{i+1, n}-v_{i, n}\right\|^{2}\right. \\
& +2 \delta_{i+1, n}\left\langle y_{i+1, n}-v_{i, n}, A_{i+1} y_{i+1, n}-A_{i+1} p\right\rangle \\
& \left.-\delta_{i+1, n}^{2}\left\|A_{i+1} y_{i+1, n}-A_{i+1} p\right\|^{2}\right] \\
& +\gamma_{i+2, n}\left[-\left\|y_{i+1, n}-w_{i, n}\right\|^{2}+2 \delta_{i+2, n}\left\langle y_{i+1, n}-v_{i, n}, A_{i+2} y_{i+1, n}-A_{i+2} p\right\rangle\right.
\end{aligned}
$$


$\left.-\delta_{i+2, n}^{2}\left\|A_{i+2} y_{i+1, n}-A_{i+2} p\right\|^{2}\right]$

$+2 \beta_{n}\left\langle P_{C}\left(I-\delta_{2, n} A_{2}\right) x_{n}-p, x_{n+1}-p\right\rangle$

$+2 \alpha_{n}\left\langle\gamma f\left(T P_{C}\left(I-\delta_{1, n} A_{1}\right) y_{1, n}\right)-F p, x_{n+1}-p\right\rangle$.

Which implies that

$$
\begin{aligned}
& \gamma_{i, n}\left\|y_{i+1, n}-u_{i, n}\right\|^{2} \\
\leq & {\left[\left\|x_{n}-p\right\|+\left\|x_{n+1}-p\right\|\right]\left\|x_{n+1}-x_{n}\right\| } \\
& +\gamma_{i, n}\left[2 \delta_{i, n}\left\langle y_{i+1, n}-u_{i, n}, A_{i} y_{i+1, n}-A_{i} p\right\rangle\right. \\
& \left.-\delta_{i, n}^{2}\left\|A_{i} y_{i+1, n}-A_{i} p\right\|^{2}\right]+\gamma_{i+1, n}\left[-\left\|y_{i+1, n}-v_{i, n}\right\|^{2}\right. \\
& \left.+2 \delta_{i+1, n}\left\langle y_{i+1, n}-v_{i, n}, A_{i+1} y_{i+1, n}-A_{i+1} p\right\rangle-\delta_{i+1, n}^{2}\left\|A_{i+1} y_{i+1, n}-A_{i+1} p\right\|^{2}\right] \\
& +\gamma_{i+2, n}\left[-\left\|y_{i+1, n}-w_{i, n}\right\|^{2}+2 \delta_{i+2, n}\left\langle y_{i+1, n}-v_{i, n}, A_{i+2} y_{i+1, n}-A_{i+2} p\right\rangle\right. \\
& \left.-\delta_{i+2, n}^{2}\left\|A_{i+2} y_{i+1, n}-A_{i+2} p\right\|^{2}\right]+2 \beta_{n}\left\langle P_{C}\left(I-\delta_{2, n} A_{2}\right) x_{n}-p, x_{n+1}-p\right\rangle \\
& +2 \alpha_{n}\left\langle\gamma f\left(T P_{C}\left(I-\delta_{1, n} A_{1}\right) y_{1, n}\right)-F p, x_{n+1}-p\right\rangle, \\
& \gamma_{i+1, n}\left\|y_{i+1, n}-v_{i, n}\right\|^{2} \\
\leq & {\left[\left\|x_{n}-p\right\|+\left\|x_{n+1}-p\right\|\right]\left\|x_{n+1}-x_{n}\right\| } \\
& +\gamma_{i, n}\left[-\left\|y_{i+1, n}-u_{i, n}\right\|^{2}+2 \delta_{i, n}\left\langle y_{i+1, n}-u_{i, n}, A_{i} y_{i+1, n}-A_{i} p\right\rangle\right. \\
& \left.-\delta_{i, n}^{2}\left\|A_{i} y_{i+1, n}-A_{i} p\right\|^{2}\right]+\gamma_{i+1, n}\left[2 \delta_{i+1, n}\left\langle y_{i+1, n}-v_{i, n}, A_{i+1} y_{i+1, n}-A_{i+1} p\right\rangle\right. \\
& \left.-\delta_{i+1, n}^{2}\left\|A_{i+1} y_{i+1, n}-A_{i+1} p\right\|^{2}\right]+\gamma_{i+2, n}\left[-\left\|y_{i+1, n}-w_{i, n}\right\|^{2}\right. \\
& \left.+2 \delta_{i+2, n}\left\langle y_{i+1, n}-v_{i, n}, A_{i+2} y_{i+1, n}-A_{i+2} p\right\rangle-\delta_{i+2, n}^{2}\left\|A_{i+2} y_{i+1, n}-A_{i+2} p\right\|^{2}\right] \\
& +2 \beta_{n}\left\langle P_{C}\left(I-\delta_{2, n} A_{2}\right) x_{n}-p, x_{n+1}-p\right\rangle \\
& +2 \alpha_{n}\left\langle\gamma f\left(T P_{C}\left(I-\delta_{1, n} A_{1}\right) y_{1, n}\right)-F p, x_{n+1}-p\right\rangle
\end{aligned}
$$

and

$$
\begin{aligned}
& \gamma_{i+2, n}\left\|y_{i+1, n}-w_{i, n}\right\|^{2} \\
\leq & {\left[\left\|x_{n}-p\right\|+\left\|x_{n+1}-p\right\|\right]\left\|x_{n+1}-x_{n}\right\| } \\
& +\gamma_{i, n}\left[2 \delta_{i, n}\left\langle y_{i+1, n}-u_{i, n}, A_{i} y_{i+1, n}-A_{i} p\right\rangle\right. \\
& \left.-\delta_{i, n}^{2}\left\|A_{i} y_{i+1, n}-A_{i} p\right\|^{2}\right]+\gamma_{i+1, n}\left[-\left\|y_{i+1, n}-v_{i, n}\right\|^{2}\right. \\
& \left.+2 \delta_{i+1, n}\left\langle y_{i+1, n}-v_{i, n}, A_{i+1} y_{i+1, n}-A_{i+1} p\right\rangle-\delta_{i+1, n}^{2}\left\|A_{i+1} y_{i+1, n}-A_{i+1} p\right\|^{2}\right] \\
& +\gamma_{i+2, n}\left[2 \delta_{i+2, n}\left\langle y_{i+1, n}-v_{i, n}, A_{i+2} y_{i+1, n}-A_{i+2} p\right\rangle\right. \\
& \left.-\delta_{i+2, n}^{2}\left\|A_{i+2} y_{i+1, n}-A_{i+2} p\right\|^{2}\right] \\
& +2 \beta_{n}\left\langle P_{C}\left(I-\delta_{2, n} A_{2}\right) x_{n}-p, x_{n+1}-p\right\rangle \\
& +2 \alpha_{n}\left\langle\gamma f\left(T P_{C}\left(I-\delta_{1, n} A_{1}\right) y_{1, n}\right)-F p, x_{n+1}-p\right\rangle .
\end{aligned}
$$


Therefore using condition $\left(B_{2}\right),(12)$ and (19) and (20) we get (16). We now show that

$$
\lim _{n \rightarrow \infty}\left\|x_{n}-T x_{n}\right\|=0
$$

Since $T$ is nonexpansive, we get

$$
\begin{aligned}
\left\|x_{n}-T x_{n}\right\| \leq & \left\|x_{n}-x_{n+1}\right\|+\left\|x_{n+1}-T P_{C}\left(I-\delta_{3, n} A_{3}\right) y_{1, n}\right\| \\
& +\left\|T P_{C}\left(I-\delta_{3, n} A_{3}\right) y_{1, n}-T P_{C}\left(I-\delta_{3, n} A_{3}\right) y_{2, n}\right\| \\
& +\left\|T P_{C}\left(I-\delta_{3, n} A_{3}\right) y_{2, n}-T y_{2, n}\right\|+\left\|T y_{2, n}-T x_{n}\right\| \\
\leq & \left\|x_{n}-x_{n+1}\right\|+\alpha_{n} \| \gamma f\left(T P_{C}\left(I-\delta_{1, n} A_{1}\right) y_{1, n}\right) \\
& -F T P_{C}\left(I-\delta_{3, n} A_{3}\right) y_{1, n}\left\|+\beta_{n}\right\| P_{C}\left(I-\delta_{2, n} A_{2}\right) x_{n} \\
& -P_{C}\left(I-\delta_{3, n} A_{3}\right) y_{1, n}\|+\| y_{1, n}-y_{2, n} \| \\
& +\left\|P_{C}\left(I-\delta_{3, n} A_{3}\right) y_{2, n}-y_{2, n}\right\|+\left\|y_{2, n}-x_{n}\right\| \\
\leq & \left\|x_{n}-x_{n+1}\right\|+\alpha_{n} \| \gamma f\left(T P_{C}\left(I-\delta_{1, n} A_{1}\right) y_{1, n}\right) \\
& -F T P_{C}\left(I-\delta_{3, n} A_{3}\right) y_{1, n} \| \\
& +\beta_{n}\left\|P_{C}\left(I-\delta_{2, n} A_{2}\right) x_{n}-P_{C}\left(I-\delta_{3, n} A_{3}\right) y_{1, n}\right\| \\
& +\left\|w_{1, n}-y_{2, n}\right\|+\sum_{i=1}^{m}\left\|y_{i, n}-y_{i+1, n}\right\|
\end{aligned}
$$
24).

Next, let us show that there exists a unique $x^{*} \in \mathcal{F}$ such that

$$
\limsup _{n \rightarrow \infty}\left\langle(F-\gamma f) x^{*}, x^{*}-x_{n}\right\rangle \leq 0 .
$$

Let $Q=P_{\mathcal{F}}$. Then $Q(I-F+\gamma f)$ is a contraction of $H$ into itself. In fact, we see that

$$
\begin{aligned}
& \|Q(I-F+\gamma f) x-Q(I-F+\gamma f) y\| \\
\leq & \|(I-F+\gamma f) x-(I-F+\gamma f) y\| \\
\leq & \|(I-F) x-(I-F) y\|+\gamma\|f(x)-f(y)\| \\
= & \lim _{n \rightarrow \infty}\left\|\left(I-\left(1-\frac{1}{n}\right) F\right) x-\left(I-\left(1-\frac{1}{n}\right) F\right) y\right\|+\gamma\|f(x)-f(y)\| \\
\leq & \lim _{n \rightarrow \infty}\left(1-\left(1-\frac{1}{n}\right) \tau\right)\|x-y\|+\gamma \alpha\|x-y\| \\
= & (1-\tau)\|x-y\|+\gamma \alpha\|x-y\|,
\end{aligned}
$$

and hence $Q(I-F+\gamma f)$ is a contraction due to $(1-(\tau-\gamma \alpha)) \in(0,1)$.

Therefore, by Banach's contraction principal, $P_{\mathcal{F}}(I-F+\gamma f)$ has a unique fixed point $x^{*}$. Then using (6), $x^{*}$ is the unique solution of the variational inequality:

$$
\left\langle(\gamma f-F) x^{*}, x-x^{*}\right\rangle \leq 0, \quad \forall x \in \mathcal{F}
$$


We can choose a a subsequence $\left\{x_{n_{j}}\right\}$ of $\left\{x_{n}\right\}$ such that

$$
\limsup _{n \rightarrow \infty}\left\langle\gamma f\left(x^{*}\right)-F x^{*}, x_{n}-x^{*}\right\rangle=\lim _{j \rightarrow \infty}\left\langle\gamma f\left(x^{*}\right)-F x^{*}, x_{n_{j}}-x^{*}\right\rangle .
$$

Because $\left\{x_{n_{j}}\right\}$ is bounded, therefore $\left\{x_{n_{j}}\right\}$ has a subsequence $\left\{x_{n_{j_{k}}}\right\}$ such that $x_{n_{j_{k}}} \rightarrow z$. Without loss of generality, we may assume that $x_{n_{j}} \rightarrow z$. it follows from (24) and Lemma 2.2 that $z \in F i x(T)$.

Now, let us show that for $i=1,2, \ldots, m+2, z \in V I\left(C, A_{i}\right)$. Let $U_{i}: H \rightarrow 2^{H}$ be a set-valued mapping defined by

$$
U_{i} x= \begin{cases}A_{i} x+N_{C} x, & x \in C, \\ \emptyset, & x \notin C,\end{cases}
$$

where $N_{C} x$ is the normal cone to $C$ at $x \in C$. Since $A_{i}$ is monotone. Thus $U$ is maximal monotone see [18]. Let $(x, y) \in G\left(U_{i}\right)$, hence $y-A_{i} x \in N_{C} x$ and since $u_{i, n}=P_{C}\left(I-\delta_{i, n} A_{i}\right) y_{i+1, n}$, therefore $\left\langle x-u_{i, n}, y-A_{i} x\right\rangle \geq 0$. On the other hand from $u_{i, n}=P_{C}\left(y_{i+1, n}-\delta_{i, n} A_{i} y_{i+1, n}\right)$, we have

$$
\left\langle x-u_{i, n}, u_{i, n}-\left(y_{i+1, n}-\delta_{i, n} A_{i} y_{i+1, n}\right)\right\rangle \geq 0
$$

that is,

$$
\left\langle x-u_{i, n}, \frac{u_{i, n}-y_{i+1, n}}{\delta_{i, n}}+A_{i} y_{i+1, n}\right\rangle \geq 0
$$

Therefore, we have

$$
\begin{aligned}
& \left\langle x-u_{i, n_{j}}, y\right\rangle \\
\geq & \left\langle x-u_{i, n_{j}}, A_{i} x\right\rangle \\
\geq & \left\langle x-u_{i, n_{j}}, A_{i} x\right\rangle-\left\langle x-u_{i, n_{j}}, \frac{u_{i, n_{j}}-y_{i+1, n_{j}}}{\delta_{i, n_{j}}}+A_{i} y_{i+1, n_{j}}\right\rangle \\
= & \left\langle x-u_{i, n_{j}}, A_{i} x-\frac{u_{i, n_{j}}-y_{i+1, n_{j}}}{\delta_{i, n_{j}}}-A_{i} y_{i+1, n_{j}}\right\rangle \\
= & \left\langle x-u_{i, n_{j}}, A_{i} x-A_{i} u_{i, n_{j}}\right\rangle+\left\langle x-u_{i, n_{j}}, A_{i} u_{i, n_{j}}-A_{i} y_{i+1, n_{j}}\right\rangle \\
& -\left\langle x-u_{i, n_{j}}, \frac{\left.u_{i, n_{j}}-y_{i+1, n_{j}}\right\rangle}{\delta_{i, n_{j}}}\right\rangle \\
\geq & \left\langle x-u_{i, n_{j}}, A_{i} u_{i, n_{j}}-A_{i} y_{i+1, n_{j}}\right\rangle-\left\langle x-u_{i, n_{j}}, \frac{\left.u_{i, n_{j}}-y_{i+1, n_{j}}\right\rangle}{\delta_{i, n_{j}}}\right\rangle \\
\geq & \left\langle x-u_{i, n_{j}}, A_{i} u_{i, n_{j}}-A_{i} y_{i+1, n_{j}}\right\rangle-\left\|x-u_{n_{j}}\right\|\left\|\frac{u_{i, n_{j}}-y_{i+1, n_{j}}}{\delta_{i, n_{j}}}\right\| .
\end{aligned}
$$

Noting that $\lim _{i \rightarrow \infty}\left\|u_{i, n_{j}}-y_{i+1, n_{j}}\right\|=0, \lim _{i \rightarrow \infty}\left\|y_{i+1, n}-x_{n}\right\|=0, x_{n_{j}} \rightarrow z$ and $A_{i}$ is $\frac{1}{\delta_{i}}$-Lipschitzian, we obtain

$$
\langle x-z, y\rangle \geq 0 \text {. }
$$

Since $U_{i}$ is maximal monotone, we have $z \in U_{i}^{-1} 0$, and hence

$$
z \in V I\left(C, A_{i}\right), \quad i=1,2, \ldots, m \text {. }
$$


Repeating the same argument as above for $v_{i, n}$ and $w_{i, n}$, we conclude that

$$
z \in V I\left(C, A_{i}\right), \quad i=2,3, \ldots, m+2 .
$$

Therefore $z \in \mathcal{F}$ and applying (27) and (28), we have

$$
\limsup _{n \rightarrow \infty}\left\langle(\gamma f-F) x^{*}, x_{n}-x^{*}\right\rangle \leq 0 \text {. }
$$

Finally, we prove that $x_{n} \rightarrow x^{*}$ as $n \rightarrow \infty$. Takeing $\tau=1-\sqrt{\frac{2-2 \delta}{1-\lambda}}$ and using (6), (11), and Lemma 2.5, we have

$$
\begin{aligned}
\left\|x_{n+1}-x^{*}\right\|^{2}= & \| \alpha_{n} \gamma f\left(T P_{C}\left(I-\delta_{1, n} A_{1}\right) y_{1, n}\right)+\beta_{n} P_{C}\left(I-\delta_{2, n} A_{2}\right) x_{n} \\
& +\left(\left(1-\beta_{n}\right) I-\alpha_{n} F\right) T P_{C}\left(I-\delta_{3, n} A_{3}\right) y_{1, n}-x^{*} \|^{2} \\
=\| & {\left[\left(\left(-\beta_{n}\right) I-\alpha_{n} F\right) T P_{C}\left(I-\delta_{3, n} A_{3}\right) y_{1, n}\right.} \\
& \left.-\left(\left(1-\beta_{n}\right) I-\alpha_{n} F\right) T P_{C}\left(I-\delta_{3, n} A_{3}\right) x^{*}\right] \\
& +\beta_{n}\left[P_{C}\left(I-\delta_{2, n} A_{2}\right) x_{n}-P_{C}\left(I-\delta_{2, n} A_{2}\right) x^{*}\right] \\
& +\alpha_{n}\left[\gamma f\left(T P_{C}\left(I-\delta_{1, n} A_{1}\right) y_{1, n}\right)-F x^{*}\right] \|^{2} \\
\leq \| & \left(\left(1-\beta_{n}\right) I-\alpha_{n} F\right) T P_{C}\left(I-\delta_{3, n} A_{3}\right) y_{n} \\
& \left.-\left(\left(1-\beta_{n}\right) I-\alpha_{n} F\right) T P_{C}\left(I-\delta_{3, n} A_{3}\right) x^{*}\right] \\
& +\beta_{n}\left[P_{C}\left(I-\delta_{2, n} A_{2}\right) x_{n}-P_{C}\left(I-\delta_{2, n} A_{2}\right) x^{*}\right] \|^{2} \\
& +2 \alpha_{n}\left\langle\gamma f\left(T P_{C}\left(I-\delta_{1, n} A_{1}\right) y_{1, n}\right)-F x^{*}, x_{n+1}-x^{*}\right\rangle \\
\leq[ & \left.\left(1-\beta_{n}-\alpha_{n} \tau\right)\left\|y_{1, n}-x^{*}\right\|+\beta_{n}\left\|x_{n}-x^{*}\right\|\right]^{2} \\
+ & 2 \alpha_{n}\left\langle\gamma f\left(P_{C}\left(I-\delta_{1, n} A_{1}\right) T y_{n}\right)-F x^{*}, x_{n+1}-x^{*}\right\rangle \\
\leq \| & {\left[\left(1-\beta_{n}-\alpha_{n} \tau\right)\left\|x_{n}-x^{*}\right\|+\beta_{n}\left\|x_{n}-x^{*}\right\|\right]^{2} } \\
& +2 \alpha_{n} \gamma\left\langle f\left(T P_{C}\left(I-\delta_{1, n} A_{1}\right) y_{1, n}\right)-f\left(x^{*}\right), x_{n+1}-x^{*}\right\rangle \\
& +2 \alpha_{n}\left\langle\gamma f\left(x^{*}\right)-F x^{*}, x_{n+1}-x^{*}\right\rangle \\
\leq(1 & \left.-\alpha_{n} \tau\right)^{2}\left\|x_{n}-x^{*}\right\|^{2}+\alpha_{n} \gamma \alpha\left[\left\|x_{n}-x^{*}\right\|^{2}+\left\|x_{n+1}-x^{*}\right\|^{2}\right] \\
+ & 2 \alpha_{n}\left\langle\gamma f\left(x^{*}\right)-F x^{*}, x_{n+1}-x^{*}\right\rangle . \\
&
\end{aligned}
$$

So we reach the following

$$
\begin{aligned}
& \left\|x_{n+1}-x^{*}\right\|^{2} \\
\leq & \frac{1+\alpha^{2} \tau^{2}-2 \alpha_{n} \tau+\alpha_{n} \gamma \alpha}{1-\alpha_{n} \gamma \alpha}\left\|x_{n}-x^{*}\right\|^{2} \\
& +\frac{2 \alpha_{n}}{1-\alpha_{n} \gamma \alpha}\left\langle\gamma f\left(x^{*}\right)-F x^{*}, x_{n+1}-x^{*}\right\rangle \\
\leq & \left(1-\alpha_{n} \frac{2(\tau-\gamma \alpha)-\alpha_{n} \tau^{2}}{1-\alpha_{n} \gamma \alpha}\right)\left\|x_{n}-x^{*}\right\|^{2} \\
& +\alpha_{n} \frac{2(\tau-\gamma \alpha)-\alpha_{n} \tau^{2}}{1-\alpha_{n} \gamma \alpha} \frac{2}{2(\tau-\gamma \alpha)-\alpha_{n} \tau^{2}}\left\langle\gamma f\left(x^{*}\right)-F x^{*}, x_{n+1}-x^{*}\right\rangle .
\end{aligned}
$$


It follows that

$$
\left\|x_{n+1}-x^{*}\right\|^{2} \leq\left(1-b_{n}\right)\left\|x_{n}-x^{*}\right\|^{2}+b_{n} c_{n},
$$

where

$$
b_{n}=\alpha_{n} \frac{2(\tau-\gamma \alpha)-\alpha_{n} \tau^{2}}{1-\alpha_{n} \gamma \alpha}
$$

and

$$
c_{n}=\frac{2}{2(\tau-\gamma \alpha)-\alpha_{n} \tau^{2}}\left\langle\gamma f\left(x^{*}\right)-F x^{*}, x_{n+1}-x^{*}\right\rangle .
$$

Since $\left\{\alpha_{n}\right\}$ satisfies in condition $\left(B_{2}\right)$, we have $\sum_{n=0}^{\infty} b_{n}=\infty$ and by condition $\left(B_{2}\right)$ and $(26)$, we get $\lim \sup _{n \rightarrow \infty} c_{n} \leq 0$. Consequently, applying Lemma 2.6, to $(29)$, we conclude that $x_{n} \rightarrow x^{*}$. Since $\left\|y_{i, n}-x^{*}\right\| \leq\left\|x_{n}-x^{*}\right\|$, for $i=1,2, \ldots, m$, we have $y_{i, n} \rightarrow x^{*}$.

By the careful analysis of the proof of Theorem 3.1, we obtain the following theorem. Because its proof is much simpler than the proof of Theorem 3.1, we omit its proof.

Theorem 3.2. Let $C$ be a closed convex subset of a real Hilbert space $H$. Let $F: C \rightarrow C$ be a mapping which is both $\delta$-strongly monotone and $\lambda$-strictly pseudo-contractive of Browder-Petryshyn type such that $\delta>(1+\lambda) / 2, f: C \rightarrow$ $C$ be a contraction with coefficient $0<\alpha<1$ and $\gamma$ be a positive real number such that $\gamma<\left(1-\sqrt{\frac{2-2 \delta}{1-\lambda}}\right) / \alpha$. Let $T: C \rightarrow C$ be a nonexpansive mapping and for each $i=1,2, \ldots, m$, let $A_{i}: C \rightarrow H$ be a $\delta_{i}$-inverse strongly monotone mapping and $\mathcal{F}=\bigcap_{i=1}^{m} V I\left(C, A_{i}\right) \cap F i x(T) \neq \emptyset$. Let $\left\{\alpha_{n}\right\}_{n=1}^{\infty}$ and $\left\{\delta_{i, n}\right\}_{i=1, n=1}^{m, \infty}$ be sequences in $(0,1)$, and $\left\{\beta_{n}\right\}_{n=1}^{\infty}$ be a sequence in $[0,1)$ satisfy the following conditions:

$\left(B_{1}\right)\left\{\delta_{i, n}\right\} \subset\left(0,2 \delta_{i}\right), i=1,2, \ldots, m$,

$\left(B_{2}\right) \lim _{n \rightarrow \infty} \alpha_{n}=0, \sum_{n=1}^{\infty} \alpha_{n}=\infty$ and $\lim _{n \rightarrow \infty} \beta_{n}=0$.

$\left(B_{3}\right) \sum_{n=1}^{\infty}\left|\alpha_{n+1}-\alpha_{n}\right|<\infty, \sum_{n=1}^{\infty}\left|\beta_{n+1}-\beta_{n}\right|<\infty, \sum_{n=1}^{\infty}\left|\delta_{i, n+1}-\delta_{i, n}\right|$ $<\infty, \quad i=1,2, \ldots, m$.

Suppose $\left\{x_{n}\right\}_{n=1}^{\infty}$ and $\left\{y_{n}\right\}_{i=1, n=1}^{m, \infty}$ are sequences generated by $x_{0} \in C$ and

$$
\begin{aligned}
& x_{n+1}=\alpha_{n} \gamma f\left(x_{n}\right)+\beta_{n} x_{n}+\left(\left(1-\beta_{n}\right) I-\alpha_{n} F\right) T P_{C}\left(I-\delta_{1, n} A_{1}\right) y_{1, n}, \\
& y_{i, n}=P_{C}\left(I-\delta_{i, n} A_{i}\right) y_{i+1, n}, \quad i=1,2, \ldots, m \\
& y_{m+1, n}=x_{n} .
\end{aligned}
$$

Then $\left\{x_{n}\right\}_{n=1}^{\infty}$ and $\left\{y_{i, n}\right\}_{i=1, n=1}^{m, \infty}$ converge strongly to $x^{*} \in \mathcal{F}$, which is the unique solution of the system of variational inequalities:

$$
\left\{\begin{array}{l}
\left\langle(F-\gamma f) x^{*}, x-x^{*}\right\rangle \geq 0, \\
\left\langle A_{i} x^{*}, x-x^{*}\right\rangle \geq 0, \quad \forall x \in \mathcal{F}, i=1,2, \ldots, m .
\end{array}\right.
$$

Corollary 3.3 (See Yao and Yao [21]). Let $C$ be a closed convex subset of a real Hilbert space $H$. Let $A$ be a $\delta$-inverse strongly monotone mapping of 
$C$ into $H$ and let $T$ be a nonexpansive mapping of $C$ into itself such that $\operatorname{Fix}(S) \cap V I(C, A) \neq \emptyset$. Suppose $x_{1}=u \in C$ and $\left\{x_{n}\right\}$ and $\left\{y_{n}\right\}$ are given by

$$
\left\{\begin{array}{l}
y_{n}=P_{C}\left(I-\lambda_{n} A\right) x_{n} \\
x_{n+1}=\alpha_{n} u+\beta_{n} x_{n}+\gamma_{n} T P_{C}\left(I-\lambda_{n} A\right) y_{n}
\end{array}\right.
$$

where $\left\{\alpha_{n}\right\},\left\{\beta_{n}\right\},\left\{\zeta_{n}\right\}$ are three sequences in $[0,1]$ and $\left\{\lambda_{n}\right\}$ is a sequence in $[0,2 \alpha]$. If $\left\{\alpha_{n}\right\},\left\{\beta_{n}\right\},\left\{\gamma_{n}\right\}$ and $\left\{\lambda_{n}\right\}$ are chosen so that $\lambda_{n} \in[a, b]$ for some $a, b$ with $0<a<b<2 \delta$ and

$$
\begin{aligned}
& \left(B_{1}\right) \alpha_{n}+\beta_{n}+\gamma_{n}=1 \\
& \left(B_{2}\right) \lim _{n \rightarrow \infty} \alpha_{n}=0, \sum_{n=1}^{\infty} \alpha_{n}=\infty \\
& \left(B_{3}\right) 0<\liminf _{n \rightarrow \infty} \lambda_{n} \leq \limsup _{n \rightarrow \infty} \lambda_{n}<1 \\
& \left(B_{4}\right) \lim _{n \rightarrow \infty}\left(\lambda_{n+1}-\lambda_{n}\right)=0 .
\end{aligned}
$$

Then $\left\{x_{n}\right\}_{n=1}^{\infty}$ converges strongly to $P_{F i x(S) \cap V I(C, A)} u$.

Proof. It suffices to take $F=I, f=\frac{1}{\gamma} u, A_{1}=A, \delta_{1, n}=\lambda_{n}, y_{1, n}=y_{n}$, $\gamma_{n}=1-\beta_{n}-\alpha_{n}$, for $n \in \mathbb{N}$ and $P_{C}\left(I-\delta_{i, n} A_{i}\right)=I$, for $i=2,3, \ldots, m$, in Theorem 3.2.

\section{References}

[1] R. P. Agarwal, D. O. Regan, and D. R. Sahu, Fixed Point Theory for Lipschitzian Type Mappings with Applications, Topological Fixed Point Theory and Its Applications, 6, Springer, New York, 2009.

[2] F. E. Browder and W. V. Petryshyn, Construction of fixed points of nonlinear mappings in Hilbert space, J. Math. Anal. Appl. 20 (1967), 197-222.

[3] L. C. Ceng, S. M. Gu, and J. C. Yao, A general iterative method with strongly positive operators for general variational inequalities, Comput. Math. Appl. 59 (2010), no. 4, 14411452.

[4] J. Chen, L. Zhang, and T. Fan, Viscosity approximation methods for nonexpansive mappings and monotone mappings, J. Math. Anal. Appl. 334 (2007), no. 2, 1450-1461.

[5] T. Jitpeera and P. Kumam, An extragradient type method for a system of equilibrium problems, variational inequality problems and fixed point of finitely many nonexpansive mappings, J. Nonlinear Anal. Optim. 1 (2010), no. 1, 71-91.

[6] _ A general iterative algorithm for generalized mixed equilibrium problems and variational inclusions approach to variational inequalities, Int. J. Math. Math. Sci. 2011 (2011), Article ID 619813, 25 pages.

[7] _ A composite iterative method for generalized mixed equilibrium problems and variational inequality problems, J. Comput. Anal. Appl. 13 (2011), no. 2, 345-361.

[8] J. S. Jung, Iterative approaches to common fixed points of nonexpansive mappings in Banach spaces, J. Math. Anal. Appl. 302 (2005), no. 2, 509-520.

[9] P. Katchang and P. Kumam, An iterative algorithm for finding a common solution of fixed points and a general system of variational inequalities for two inverse strongly accretive operators, Positivity 15 (2011), no. 2, 281-295.

[10] G. Marino and H. K. Xu, A general iterative method for nonexpansive mappings in Hilbert spaces, J. Math. Anal. Appl. 318 (2006), no. 1, 43-52.

[11] M. O. Osilike and D. I. Igbokwe, Weak and strong convergence theorems for fixed points of pseudocontractions and solutions of monotone type operator equations, Comput. Math. Appl. 40 (2000), no. 4-5, 559-567. 
[12] H. Piri, A general iterative method for finding common solutions of system of equilibrium problems, system of variational inequalities and fixed point problems, Math. Comput. Modell. 55 (2012), 1622-1638.

[13] Solutions of variational inequalities on fixed points of nonexpansive mappings, Bull. Iranian Math. Soc. 39 (2013), no. 4, 743-764.

[14] - Hybrid pseudo-viscosity approximation schemes for systems of equilibrium problems and fixed point problems of infinite family and semigroup of non-expansive mappings, Nonlinear Anal. 74 (2011), no. 17, 6788-6804.

[15] - Strong convergence for a minimization problem on solutions of systems of equilibrium problems and common fixed points of an infinite family and semigroup of nonexpansive mappings, Comput. Math. Appl. 61 (2011), no. 9, 2562-2577.

[16] H. Piri and A. H. Badali, Strong convergence theorem for amenable semigroups of nonexpansive mappings and variational inequalities, Fixed Point Theory Appl. 2011 (2011), doi:10.1186/1687-1812-2011-55.

[17] S. Plubtieng and R. Wangkeeree, Strong convergence theorems for three-step iterations with errors for non-lipschitzian nonself-mappings in Banach spaces, Comput. Math. Appl. 51 (2006), no. 6-7, 1093-1102.

[18] R. T. Rockafellar, On the maximality of sums of nonlinear monotone operators, Trans. Amer. Math. Soc. 149 (1970), 75-88.

[19] N. Xiu, Y. Wang, and X. Zhang, Modified fixed-point equations and related iterative methods for variational inequalities, Comput. Math. Appl. 47 (2004), no. 6-7, 913-920.

[20] H. K. Xu, An iterative approach to quadratic optimization, J. Optim. Theory Appl. 116 (2003), no. 3, 659-678.

[21] Y. Yao and J. C. Yao, On modified iterative method for nonexpansive mappings and monotone mappings, Appl. Math. Comput. 186 (2007), no. 2, 1551-1558.

Department of MATHEMATics

UNIVERSITY OF BONAB

BONAB 55517-61167, IRAN

E-mail address: hossein_piri1979@yahoo.com; h.piri@bonabetu.ac.ir 